\title{
HaCaT-conditioned medium supplemented with the small molecule inhibitors SB431542 and CHIR99021 and the growth factor PDGF-AA prevents the dedifferentiation of dermal papilla cells in vitro
}

\author{
DONGJIE SUN ${ }^{1}$, ZHEHAO HUANG $^{2}$, JINYING XU $^{1}$, YIQING WANG ${ }^{3}$, \\ LIN CHEN $^{1,4}$, YI HOU ${ }^{5}$ and GUANGFAN CHI ${ }^{1}$ \\ ${ }^{1}$ The Key Laboratory of Pathobiology, Ministry of Education, College of Basic Medical Sciences, \\ Jilin University; ${ }^{2}$ Department of Neurosurgery, China-Japan Union Hospital of Jilin University; \\ ${ }^{3}$ Department of Genetics, Basic Medical College of Jilin University; ${ }^{4}$ Department of Gastroenterology, \\ China-Japan Union Hospital of Jilin University; ${ }^{5}$ Department of Regeneration Medicine, \\ School of Pharmaceutical Science of Jilin University, Changchun, Jilin 130000, P.R. China
}

Received June 3, 2020; Accepted December 11, 2020

DOI: $10.3892 / \mathrm{mmr} .2021 .11965$

\begin{abstract}
Hair loss, including alopecia, is a common and distressing problem for men and women, and as a result, there is considerable interest in developing treatments that can prevent or reverse hair loss. Dermal papillae closely interact with epidermal cells and play a key role during hair follicle induction and hair morphogenesis. As dermal papilla cells (DPCs) lose their hair-inducing ability in monolayer cultures in vitro, it is difficult to obtain de novo hair follicle structures following DPC transplantation in vivo. The present study aimed to explore culture conditions to maintain DPC characteristics using conditioned media (CM) from the supernatant of cultured HaCaT keratinocyte cells supplemented with other components. Initially, it was observed that during passaging of in vitro monolayer DPC cultures, the $\mathrm{Wnt} / \beta$-catenin pathway was repressed, while the TGF- $\beta /$ Smad pathway was activated, and that $\mathrm{HaCaT}$ cells cultivated in $1 \%$ fetal bovine serum had higher levels of expression of Wnt $3 \mathrm{a}$ and Wnt10b compared with normal keratinocytes. Culturing of high-passage (P7) DPCs in CM from HaCaT cells (HaCaT-CM) actively stimulated cell proliferation and maintained Sox 2 and Versican expression levels. Supplementation of HaCaT-CM with SB431542 (SB, a TGF- $\beta$ receptor inhibitor), CHIR99021, (CHIR, a GSK3 $\alpha / \beta$
\end{abstract}

Correspondence to: Professor Guangfan Chi, The Key Laboratory of Pathobiology, Ministry of Education, College of Basic Medical Sciences, Jilin University, 126 Xinmin Street, Changchun, Jilin 130000, P.R. China

E-mail: guangfan130@jlu.edu.cn

Key words: dermal papilla cells, dedifferentiation, HaCaT cells, small molecule inhibitors, hair follicle regeneration, three-dimensional culture inhibitor and activator of Wnt signaling) and platelet-derived growth factor (PDGF)-AA further increased the expression levels of Sox 2, Versican and alkaline phosphatase (ALP) in P7 DPCs. Three-dimensional culture of P7 DPCs using hanging drop cultures in HaCaT-CM supplemented with SB, CHIR and PDGF-AA resulted in larger cell aggregates and a further significant upregulation of Sox2, ALP and Versican expression levels. Taken together, these findings demonstrated that HaCaT-CM supplemented with SB, CHIR and PDGF-AA may preserve the hair-inducing ability of high-passage DPCs and may therefore be useful in reconstructing new hair follicles in vivo.

\section{Introduction}

Hair loss, including alopecia, is a common and distressing problem for men and women, and as a result there is considerable interest in developing treatments that can prevent or reverse this hair loss. Currently, treatment with drugs and hair transplantation are the conventional treatments for hair loss or alopecia. However, drug treatments only delay hair loss and cannot prevent further hair loss (1). While autologous hair follicle transplantation is an effective treatment for hair loss, the area for extracting intact hair follicles (HFs) is limited in a patient's scalp, and so the available number of hair follicles that can be extracted is also limited $(1,2)$. Thus, hair follicle regeneration through bioengineering is now considered to be a promising alternative strategy for treating hair loss.

In scalp tissue, dermal papilla (DP) cells (DPCs), which are derived from neural crest stem cells, are specialized fibroblast-like cells and are hypothesized to play crucial roles in hair follicle morphogenesis and cycling $(3,4)$. Therefore, DPCs are essential for the successful construction of bioengineered HFs to treat hair loss. Although freshly dissociated fully intact DPCs have hair-inducing characteristics, they rapidly lose this capability following serial sub-cultivation due 
to a process of cell dedifferentiation, which is characterized by changes in their morphology and a downregulation of their characteristic genes (5-9). As such, obtaining a large number of DPCs through modification of ex vivo culture conditions remain a challenge.

In vivo, DPCs congregate and form a three-dimensional (3D) DP structure, which is located at the base of the hair follicle bulb and are surrounded by a dermal sheath and the hair matrix (7). In addition to DPCs, other cellular components of the hair bulb include keratinocytes and melanocytes (5). Thus, in the bulb, DPCs are closely in contact with each other and exist in a specialized 3D environment. Furthermore, their cellular properties are strictly controlled by complex elements, including cell-cell interactions, the extracellular matrix (ECM) and various factors, such as growth factors and cytokines that are secreted by DPCs and surrounding cells $(2,10)$. According to reports, the DPC phenotype is tightly regulated by various signaling pathways, including the Wnt/ $\beta$-catenin, platelet-derived growth factor (PDGF) and bone morphogenetic protein (BMP) pathways (11-15). In particular, DPCs and keratinocytes interact with each other in a reciprocal manner, suggesting that signals from keratinocytes are vital for maintaining the biological function of DPCs (2). Therefore, during in vitro culture, culture conditions that mimic the in vivo environment should be used in order to provide a more favorable environment that is able to preserve DPC trichogenicity.

The present study explored new culture conditions using conditioned media (CM) derived from the supernatant of cultured HaCaT cells supplemented with SB431542 (SB, an inhibitor of the TGF $\beta /$ Smad pathway), CHIR99021 (CHIR, a GSK $3 \alpha / \beta$ inhibitor and activator of Wnt signaling), and PDGF-AA. Using this media, high-passage (P7) DPCs were cultured under both two-dimensional (2D) and 3D culture conditions and changes in morphology and gene expression patterns associated with the trichogenic phenotype of DPCs were examined.

\section{Materials and methods}

Isolation of DPCs and cell culture. Full-thickness skin samples were obtained from the occipital human scalp of three individuals undergoing corrective surgery for the treatment of androgenetic alopecia. The experimental protocol was established according to the ethical guidelines of the Helsinki Declaration and was approved by the Human Ethics Committee of China-Japan Union Hospital of Jilin University (approval no. 2020042606; Changchun, China). Written informed consent was obtained from individual patients. Follicles were removed from the fine scalp. Collagen capsules surrounding the scalp follicles were then removed to expose the follicle bases, and DPs were dissected using thin needles. Isolated DPs were placed on the bottom of the cell culture dishes. DPCs were cultured for 10-14 days, harvested using $0.25 \%$ trypsin-EDTA (Sigma-Aldrich; Merck KGaA), and transferred to fresh culture dishes. DPCs were cultured in DMEM-F12 (Gibco; Thermo Fisher Scientific, Inc.) supplemented with $10 \%$ fetal bovine serum (FBS; Gibco; Thermo Fisher Scientific, Inc.) and fibroblast growth factor-basic (bFGF; $10 \mathrm{ng} / \mathrm{ml}$; PeproTech, Inc.). In total, $4 \times 10^{4}$ cells $/ \mathrm{ml}$ DPCs were cultured in $\mathrm{T} 75$ culture flasks. Cells were subcultured or harvested upon reaching
$80-90 \%$ confluence. The culture medium was exchanged every three days. The cells were examined under a bright-field microscope (magnification, x40) (Olympus Corporation), the cell aspect ratio (measured as the length of the long axis divided by that of the narrow axis) and cell areas were analyzed using cellSens Dimension software (version 1.12; Olympus Corporation).

Keratinocyte culture. The foreskin of children (discarded tissue after circumcision) was obtained from China-Japan Union Hospital of Jilin University. Procedures were explained and written informed consent was obtained from participants' guardians in accordance with Declaration of Helsinki guidelines. The experimental procedures were officially approved by the Human Ethics Committee as described above (approval no. 2020042606). The epidermis was separated from the foreskin after overnight incubation with $2 \mathrm{~g} / \mathrm{ml}$ dispase II (Gibco; Thermo Fisher Scientific, Inc.) at $4^{\circ} \mathrm{C}$, and keratinocytes were isolated after trypsinization for $7 \mathrm{~min}$ by thorough pipetting. Keratinocytes were cultured at $37^{\circ} \mathrm{C}$ in Epidermal Keratinocyte Medium (Gibco; Thermo Fisher Scientific, Inc.). After cells reached $90 \%$ confluence, the medium was completely replaced with DMEM-F12 supplemented with $1 \%$ FBS (v/v) and cultured for $24 \mathrm{~h}$, followed by harvesting the media for further analysis.

HaCaT cell culture and HaCaT-CM preparation. HaCaT cells were obtained from The Cell Bank of Type Culture Collection of the Chinese Academy of Sciences. Cells were cultured to $80-90 \%$ confluence in T75 culture flasks and treated with $10 \%$ FBS (v/v). Following this, the medium was replenished with $10 \mathrm{ml}$ DMEM-F12 supplemented with different concentrations of FBS $\left(0,1\right.$ and $10 \%$ by volume) at $37^{\circ} \mathrm{C}$. For HaCaT-CM preparation, the cells were cultured at $37^{\circ} \mathrm{C}$ for 24 and $48 \mathrm{~h}$ with $1 \% \mathrm{FBS}(\mathrm{v} / \mathrm{v})$ before harvesting. The collected supernatant samples were filtered through a $0.2-\mu \mathrm{m}$ filter and stored at $-20^{\circ} \mathrm{C}$. Then, the supernatant was used to culture DPCs at different concentrations $(0,25,50$ and $100 \%$ by volume).

Alkaline phosphatase (ALP) activity. ALP levels were assessed using the BCIP/NBT Alkaline Phosphatase Color Development Kit (Beyotime Institute of Biotechnology), according to the manufacturer's instructions. Human DPCs were plated in 12-well plates at 8x104 cells per well. After growing for $24 \mathrm{~h}$, the cells were fixed in $4 \%$ paraformaldehyde at room temperature for $10 \mathrm{~min}$ and then washed with phosphate-buffered saline (PBS). Cells were then incubated in BCIP/NBT buffer at room temperature for $30 \mathrm{~min}$. The reaction was stopped by washing with PBS, and the cells were examined under a bright-field microscope (magnification, $\mathrm{x} 40$ ). Dark blue staining indicated a positive signal for ALP.

Reverse transcription $(R T)$-polymerase chain reaction $(P C R)$ and $R T$-quantitative (q)PCR analyses. To examine the expression of genes in DPCs, total RNA $(1 \mu \mathrm{g})$ was isolated using TRIzol $^{\circledR}$ reagent (Invitrogen; Thermo Fisher Scientific, Inc.), and first-strand cDNA was synthesized using SuperScript ${ }^{\mathrm{TM}}$ III Reverse Transcriptase (Invitrogen; Thermo Fisher Scientific, Inc.) and oligo-dT (Promega Corporation), according to the manufacturer's instructions. For RT-PCR, the amplification 
included initial denaturation at $95^{\circ} \mathrm{C}$ for $10 \mathrm{sec}, 35$ cycles of denaturation at $95^{\circ} \mathrm{C}$ for $10 \mathrm{sec}$, and annealing at $60^{\circ} \mathrm{C}$ for $30 \mathrm{sec}$. The GAPDH mRNA level was used for sample standardization. The PCR products were separated by electrophoresis on a 1.5\% agarose gel (Beyotime Institute of Biotechnology) and were stained with GelRed ${ }^{\circledR}$ (Beyotime Institute of Biotechnology), each band was quantified using ImageJ 1.53a software (National Institutes of Health). For RT-qPCR, a TransStart Tip Green qPCR SuperMix (Beijing TransGen Biotech Co., Ltd.) was used, and the thermocycling program was as follows: $94^{\circ} \mathrm{C}$ for $30 \mathrm{sec}$, followed by 40 cycles at $94^{\circ} \mathrm{C}$ for $5 \mathrm{sec}, 55^{\circ} \mathrm{C}$ for $15 \mathrm{sec}$ and $72^{\circ} \mathrm{C}$ for $10 \mathrm{sec}$. At least three independent biological replicates were performed for the RT-qPCR. The amount of PCR product was calculated relative to the internal control $G A P D H$, and then compared between the experimental and control groups using the $2^{-\Delta \Delta \mathrm{Cq}}$ method (16). Primer sequences were as follows: Sox 2 sense, 5'-CGGATTATAAATACCGGC CC-3' and antisense, 5'-GTG TACTTATCCTTCATGAGC-3'; ALP sense, 5'-CAGGTC CCACAAGCCCGCAA-3' and antisense, 5'-CCCGGTGGG CCACAAAA-3'; Versican sense, 5'-TGAGCATGACTTCCG TTGGACTGA-3' and antisense, 5'-CCACTGGCCATTCTC ATGCCAAAT-3'; Wnt3a sense, 5'-AGATTGGCATCCAGG AGTG-3' and antisense, 5'-CTCCCTGGTAGCTTTGTCC-3'; Wnt10a sense, 5'-CTAAGGACTTTCTGGACTCCC-3' and antisense, 5'-TGTTCTCCATCACTGCCTG-3'; Wnt10b sense, 5'-GAGGTCCTGATCGATCTGC-3' and antisense, 5'-ATT GCTTAGAGCCCGACTG-3'; EGF sense, 5'-GAGAAACTG TTGGGAGAGGAATC-3' and antisense, 5'-TCACAGAGT TTAACAGCCCTGC-3'; BMP6 sense, 5'-TCAGCACAGAGA CTCTGAC-3' and antisense, 5'-ATGTCAAATTCCAGCCA GC-3'; GAPDH sense, 5'-CGCTCTCTGCTCCTGTT-3' and antisense, 5'-CCATGGTGTCTGAGCGATGT-3'.

Western blot analysis. Cells were lysed in RIPA buffer [150 mM NaCl, 10 mM Tris, $\mathrm{pH}$ 7.2, 0.1\% sodium dodecyl sulfate (SDS), $1.0 \%$ Triton X-100, 1\% sodium deoxycholate, $5 \mathrm{mM}$ EDTA]. Protein concentrations were determined by the Bradford assay (Beyotime Institute of Biotechnology). Samples (25 $\mu \mathrm{g} /$ lane) were separated via SDS-PAGE on 10-12\% gels, following which, proteins were electrophoretically transferred onto a nitrocellulose membrane. After blocking in sterile PBS containing $5 \%$ non-fat dry skimmed milk and $0.05 \%(\mathrm{v} / \mathrm{v})$ Tween-20 at room temperature for $1 \mathrm{~h}$, the blots were incubated with the following primary antibodies overnight at $4^{\circ} \mathrm{C}$ : Sox 2 (cat. no. 3579; 1:1,000; Cell Signaling Technology, Inc.), ALP (cat. no. ab108337; 1:2,000; Abcam), Versican (cat. no. ab19345; 1:1,000; Abcam), phosphorylated (p)-Smad2/3 (cat. no. 8828; 1:1,000; Cell Signaling Technology, Inc.), Smad2/3 (cat. no. 5678; 1:1,000; Cell Signaling Technology, Inc.), $\beta$-catenin (cat. no. 8480; 1:1,000; Cell Signaling Technology, Inc.), lymphoid enhancer-binding factor 1 (Lef-1) (cat. no. 2230; 1:1,000; Cell Signaling Technology, Inc.) and $\beta$-actin (cat. no. AT0001; 1:1,000; Engibody Biotechnology, Inc.). Horseradish peroxidase-conjugated anti-mouse (cat. no. 7076; 1:1,000; Cell Signaling Technology, Inc.) and anti-rabbit (cat. no. 7074; 1:1,000; Cell Signaling Technology, Inc.) antibodies were used as secondary antibodies at room temperature for $1 \mathrm{~h}$. The immune complexes were assayed with an enhanced chemiluminescence kit (Invitrogen; Thermo
Fisher Scientific, Inc.) and the blots were analyzed with densitometry using ImageJ 1.53a software (National Institutes of Health).

Cell proliferation assay. The cell proliferation assay was carried out using a Cell Counting Kit-8 (CCK-8) cell proliferation assay kit (Dojindo Molecular Technologies, Inc.) according to the manufacturer's instruction. DPCs were placed into 96-well plates $\left(2 \times 10^{3}\right.$ cells/well). After culturing for 1,2 , $3,4,5,6$ or 7 days, $10 \mu$ l CCK-8 assay solution was added to each well. Subsequently, after incubation for $2 \mathrm{~h}$, the optical density (OD) at $450 \mathrm{~nm}$ was measured with an enzyme immunoassay analyzer (Thermo Fisher Scientific, Inc.) to estimate cell proliferation.

Histological and immunocytochemical analyses. DPC spheres were harvested for histological and immunocytochemical analyses. The treated spheres were fixed in $4 \%$ paraformaldehyde at room temperature for $24 \mathrm{~h}$, dehydrated with sucrose and embedded in optimum cutting temperature compound. The frozen sections $(6-\mu \mathrm{m}$ thick) were visualized using hematoxylin and eosin (H\&E) stain at room temperature and observed under a bright-field microscope (Olympus Corporation). For immunocytochemical analysis, cells were washed with PBS, fixed in $4 \%$ paraformaldehyde at room temperature for $30 \mathrm{~min}$, and incubated in PBS for $20 \mathrm{~min}$ at $4^{\circ} \mathrm{C}$. Subsequently, the cells were permeabilized using $0.1 \%$ Triton X-100 for $15 \mathrm{~min}$, followed by blocking with 5\% FBS at room temperature for $30 \mathrm{~min}$. The frozen sections were incubated with ALP (cat. no. ab108337; 1:100; Abcam) and Versican (cat. no. ab19345; 1:100; Abcam) or Sox2 (cat. no. 3579; 1:100; Cell Signaling Technology, Inc.) at $4^{\circ} \mathrm{C}$ overnight. The sections were then washed in PBS with $0.05 \%$ (v/v) Tween-20 and incubated with Alexa Fluor ${ }^{\circledR}$ 594-conjugated goat anti-rabbit IgG (cat. no. R37117; 1:200; Invitrogen; Thermo Fisher Scientific, Inc.) at room temperature for $1 \mathrm{~h}$. The frozen sections were stained with DAPI (1:2,000; Sigma-Aldrich; Merck KGaA) at room temperature for $10 \mathrm{~min}$ and observed via fluorescence microscopy (Olympus Corporation).

Hanging drop aggregation assay. DPCs at P7 (3x10 3 cells/ml; $40 \mu \mathrm{l})$ were disseminated and pipetted into each well of the 96-well hanging drop dishes (Sigma-Aldrich; Merck KGaA). The medium for the HaCaT-CM + 3D group was HaCaT-CM supplemented with $10 \mathrm{mM} \mathrm{SB}$ (TGF- $\beta$ receptor inhibitor; Tocris Bioscience), $3 \mathrm{mM} \mathrm{CHIR} \mathrm{(GSK3} \mathrm{inhibitor} \mathrm{and} \mathrm{activator}$ of the Wnt pathway; Peprotech, Inc.), and $5 \mathrm{ng} / \mathrm{ml}$ growth factor PDGF-AA (Peprotech, Inc.). The medium of the control group was DMEM-F12 supplemented with $1 \%$ FBS (v/v). Culture media were changed every $24 \mathrm{~h}$. The cultures were placed at $37^{\circ} \mathrm{C}$ in a humidified incubator with a $5 \% \mathrm{CO}_{2}$ atmosphere. Subsequently, the cells from both groups were harvested throughout the culture period of 5 days and evaluated in terms of morphology and gene expression. The diameter and surface area of DPC spheres were analyzed using the cellSens Dimension software (version 1.12; Olympus Corporation).

Statistics. The results are expressed as the mean \pm SEM. All experiments were repeated three times with independent cultures, and similar results were obtained. Statistical 

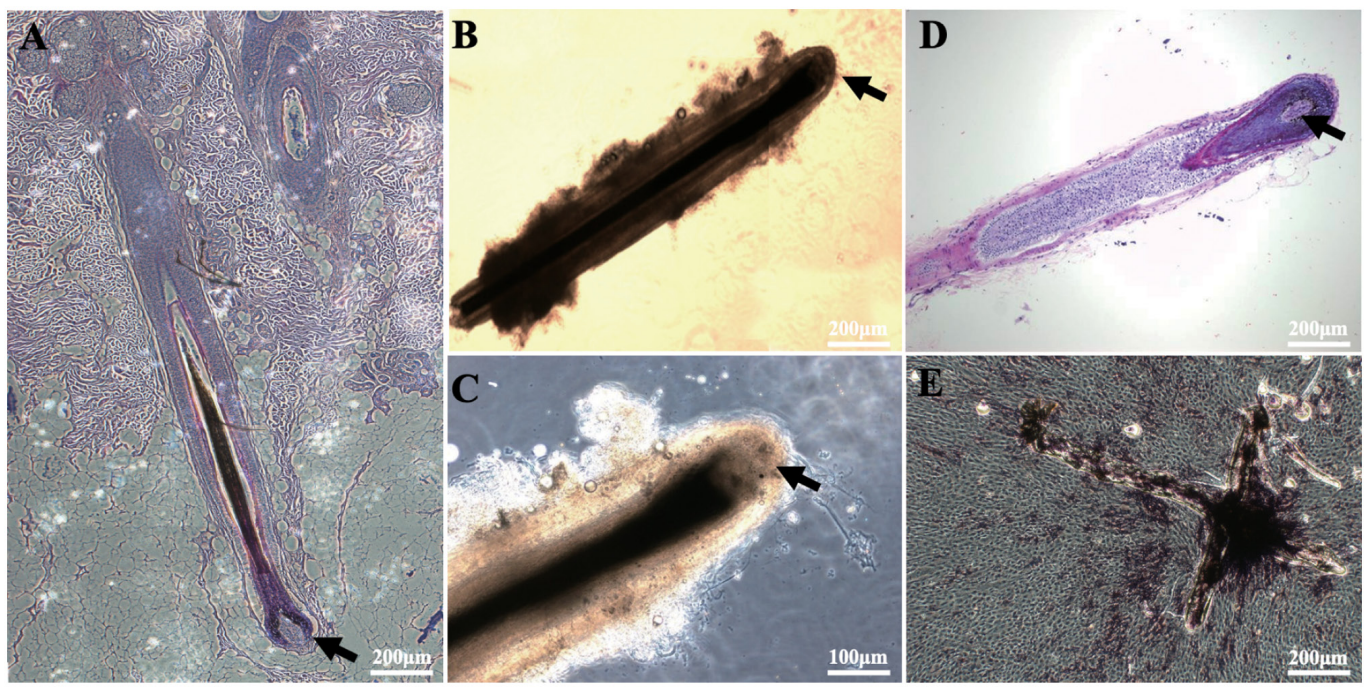

Figure 1. Isolation of human DPs and ALP staining. (A) H\&E staining of human hair follicles in the anagen phase; arrowheads indicate DP (scale bar, $200 \mu \mathrm{m}$ ). (B and C) Structure of DP by optical microscopy (scale bars, 200 or $100 \mu \mathrm{m}$ ). (D) H\&E staining of DP (scale bar, $200 \mu \mathrm{m}$ ). (E) ALP staining of cultured DP and DPCs (scale bar, $200 \mu \mathrm{m}$ ). DP, dermal papilla; DPC, dermal papilla cells; H\&E, hematoxylin and eosin; ALP, alkaline phosphatase.

A

P1

P3

P5

P7
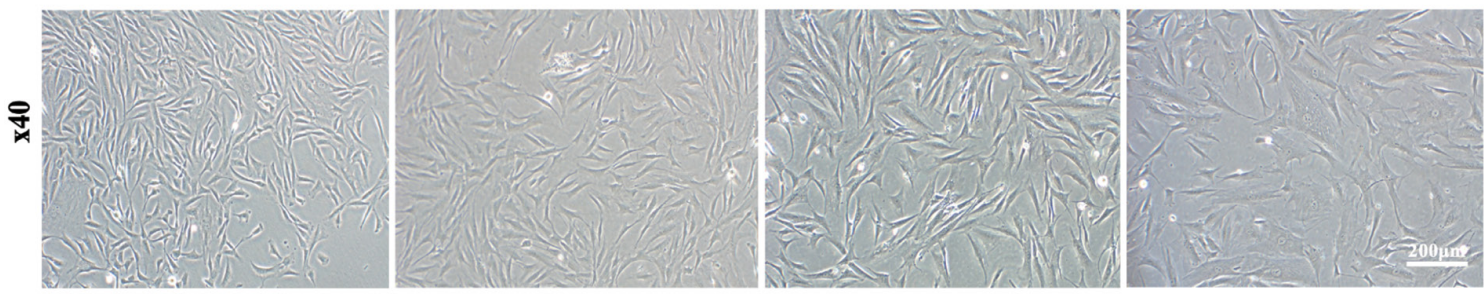

B
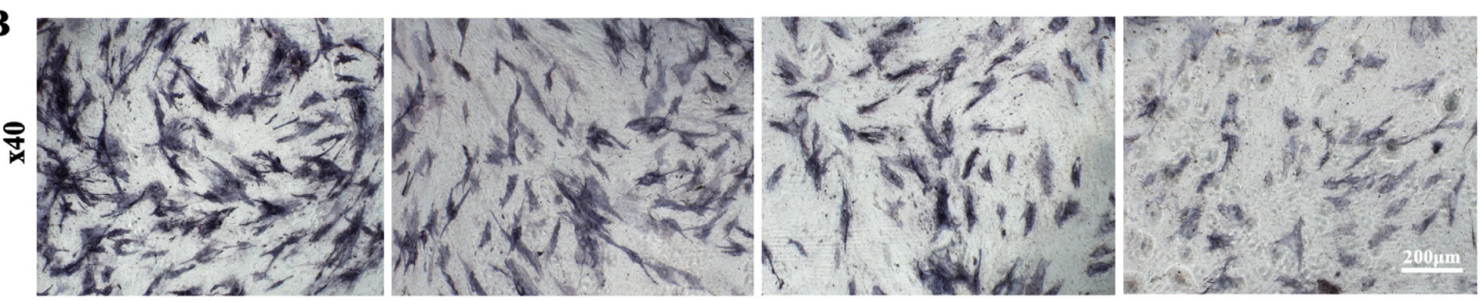

C

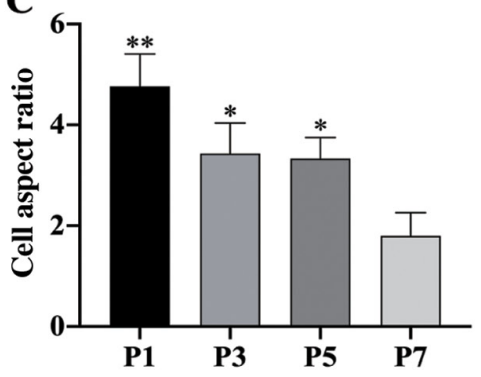

D

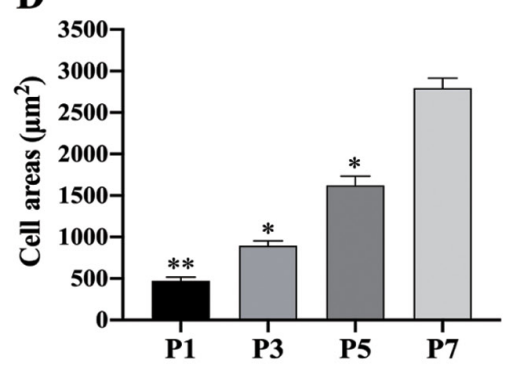

$\mathbf{E}$

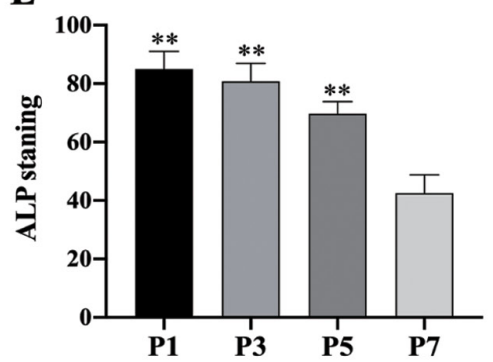

Figure 2. Morphological observation and specific ALP staining of DPCs at different passages. (A) Morphology of DPCs at passages P1, P3, P5 and P7 (scale bar, $200 \mu \mathrm{m}$ ). (B) ALP staining of DPCs at passages P1, P3, P5 and P7 (scale bar, $200 \mu \mathrm{m}$ ). (C) Cell aspect ratio of DPCs at P1, P3, P5 and P7; n=20 in each group. (D) Cell areas of DPCs at passages P1, P3, P5 and P7; n=20 in each group. (E) ALP staining of DPCs at passages P1, P3, P5 and P7; n=20 in each group. Data represent the mean $\pm \mathrm{SEM} .{ }^{*} \mathrm{P}<0.05,{ }^{* *} \mathrm{P}<0.01$ vs. P7 DPCs. DPC, dermal papilla cells; ALP, alkaline phosphatase; $\mathrm{P}$, passage.

tests were carried out with SPSS (version 22.0; SPSS, Inc.). Statistical significance was determined using one-way analysis of variance (ANOVA), followed by Tukey's post hoc test. For comparisons between two treatment groups unpaired Student's t-test was used. $\mathrm{P}<0.05$ was considered to indicate a statistically significant difference.

\section{Results}

Isolation of human DPs and ALP staining. First, HFs were separated, and their morphology was examined under a microscope following histochemical staining. In addition, DPCs (from micro-dissected DPs) were characterized by ALP 
A

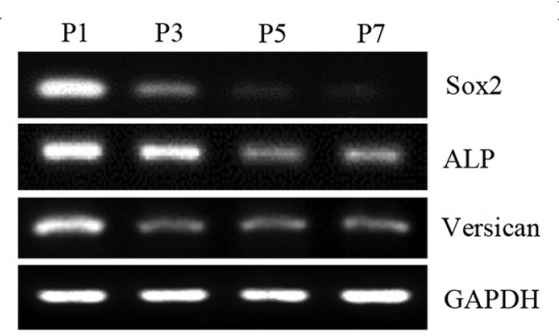

B

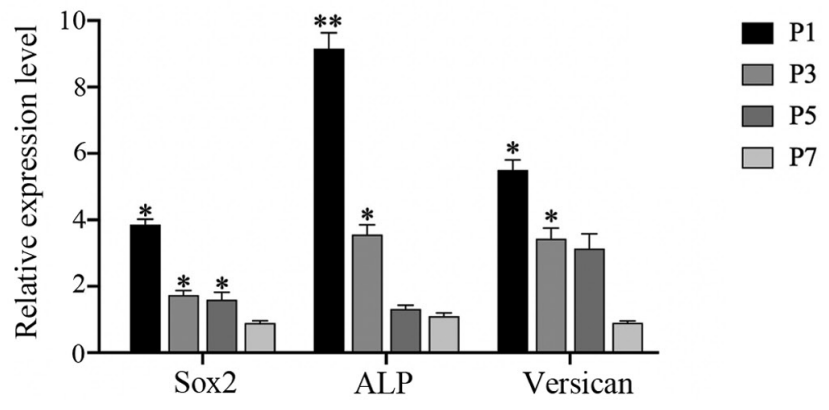

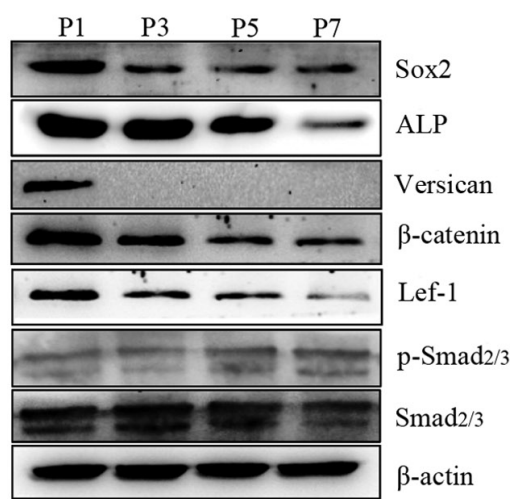

$\mathrm{D}$

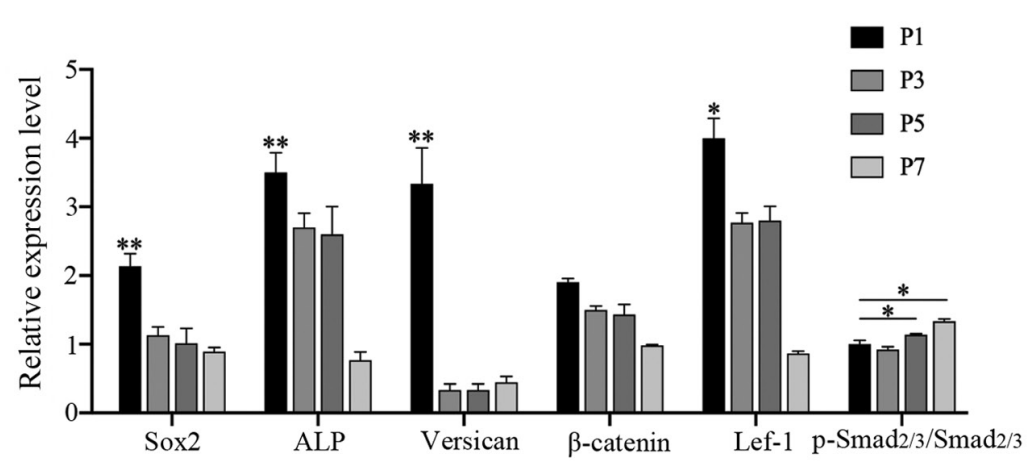

Figure 3. Expression of characteristic genes and proteins in DPCs during subculture. (A) RT-PCR analysis of Sox2, ALP and Versican mRNA levels in DPCs (P1, P3, P5, P7) subcultured in control medium (DMEM-F12 supplemented with basic fibroblast growth factor). (B) RT-qPCR analysis of Sox2, ALP and Versican mRNA levels in DPCs (P1, P3, P5, P7) in control medium; $n=3$. (C) Western blot analysis of Sox2, ALP, Versican, $\beta$-catenin, Lef-1, total Smad2/3 and $p-S m a d 2 / 3$ levels in DPCs (P1, P3, P5, P7) in control medium; (D) Densitometry analysis; $n=3$. Data represent the mean \pm SEM. ${ }^{*} \mathrm{P}<0.05$, ${ }^{* * *} \mathrm{P}<0.01$ vs. P7 DPCs. DPC, dermal papilla cells; ALP, alkaline phosphatase; P, passage; RT-PCR, reverse transcription-quantitative PCR; RT-qPCR, RT-quantitative PCR; Lef-1, lymphoid enhancer-binding factor 1; p-, phosphorylated.

staining. The results showed that DPs were located in the hair bulb at the bottom of the HFs (Fig. 1A and B), and that they had a very high cell density in the follicular growth phase (Fig. $1 \mathrm{C}$ and D). Next, DPs were isolated from the HFs and placed in dishes, after which the DPCs were observed to spread out and underwent proliferation after $\sim 14$ days (Fig. 1E). It was also observed that there was high ALP activity in the cultured DPs and DPCs (Fig. 1E).

Morphological observation and specific ALP staining of subcultured DPCs. Upon observing the subcultured DPCs, the morphology of DPCs was found to change notably during subculture. Specifically, the morphology of DPCs gradually shifted from long-spindle shaped cells to polygonal shaped cells at higher cell generations (Fig. 2A). Furthermore, the amount of ALP-stained cells decreased over the culture period (Fig. 2B and E). A statistical analysis showed that the aspect ratio of the cells decreased at higher cell generations, while the cell surface area gradually increased (Fig. 2C and D).

Expression of specific genes and proteins in DPCs during subculture. Changes in the expression of hair induction-related genes and proteins were examined during DPC culture. The results showed that the expression levels of hair-inducing genes Sox2, ALP and Versican were decreased significantly compared with P1-DPCs (Fig. 3A and B). Western blotting was used to measure the protein expression levels of DPCs during subculture, including the hair-inducing proteins Sox2, ALP and Versican, the Wnt pathway proteins $\beta$-catenin and
Lef-1, and the TGF- $\beta /$ Smad pathway protein $\mathrm{p}-\mathrm{Smad} 2 / 3$. The results showed that Sox2, ALP and Versican expression levels decreased rapidly during subsequent passaging. Moreover, the expression levels of $\beta$-catenin and Lef- 1 also gradually decreased, while the levels of $\mathrm{p}-\mathrm{Smad} 2 / 3$ gradually increased with passaging (Fig. 3C and D).

Preparation and evaluation of $\mathrm{HaCaT-CM}$. HaCaT cells were cultured with different concentrations of FBS (0, 1 and 10\% by volume) and the gene expression levels of a number of key genes were measured. HaCaT cells cultured in $0 \% \mathrm{FBS}$ were found to grow more slowly than those cultured in $1 \%$ or $10 \%$ FBS. Cells cultured in the presence of $1 \%$ and $10 \%$ FBS showed no notable differences in growth rate and morphology (Fig. 4A). Keratinocytes were also isolated and cultured (Fig. 4B). HaCaT cells also showed higher mRNA expression levels of Wnt3a, Wnt10a, Wnt10b and EGF than DPCs (Fig. 4C and D), as well as higher expression levels of Wnt3a and $W n t 10 b$ than primary keratinocytes isolated from human foreskin (Fig. 4E and F). To reduce the interference of exogenous serum cytokines, the supernatant of HaCaT cells cultured with $1 \% \mathrm{FBS}$ at different time points (24 or $48 \mathrm{~h}$ ) were collected. Subsequently, the supernatant was used to culture DPCs at different concentrations (0, 25, 50 and 100\%). Cell proliferation and gene expression of the DPCs in each group were measured and it was found that DPCs had a higher growth rate and higher expression levels of Sox 2 and Versican in a $24 \mathrm{~h}$ culture with a $50 \%$ volume fraction of the supernatant (Fig. 4G and H). As such, the supernatant under this condition was designated as HaCaT-CM. 


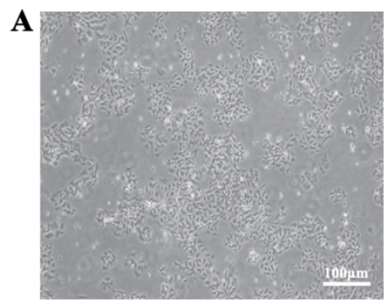

0\% FBS

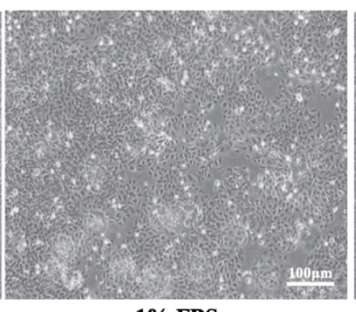

$1 \%$ FBS

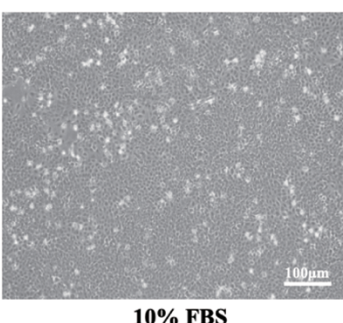

$10 \%$ FBS

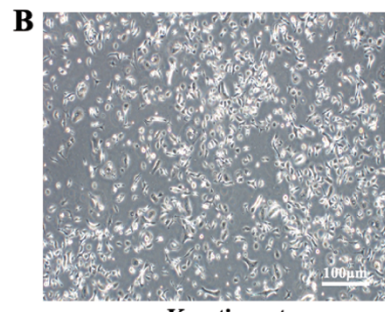

Keratinocyte
C

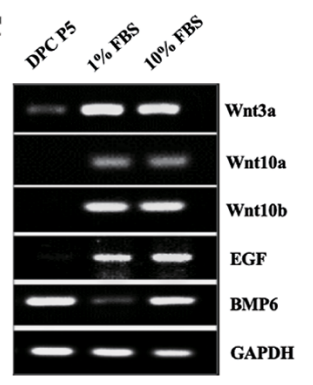

D

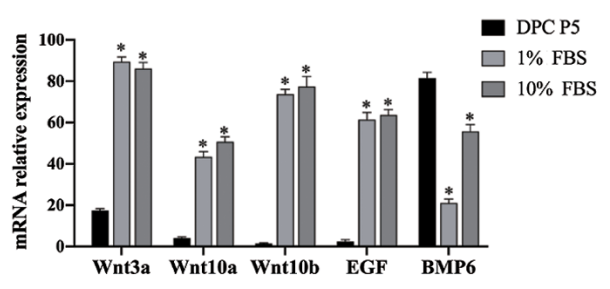

G
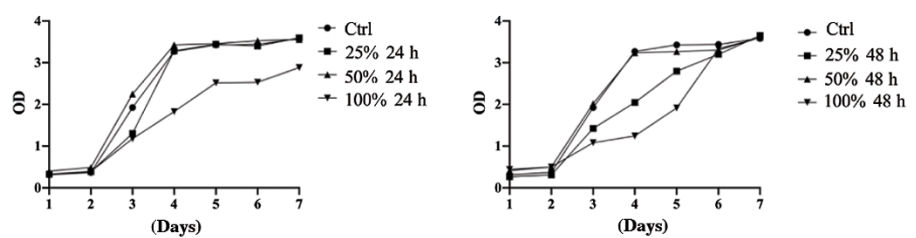

$\mathbf{E}$

$\mathbf{F}$
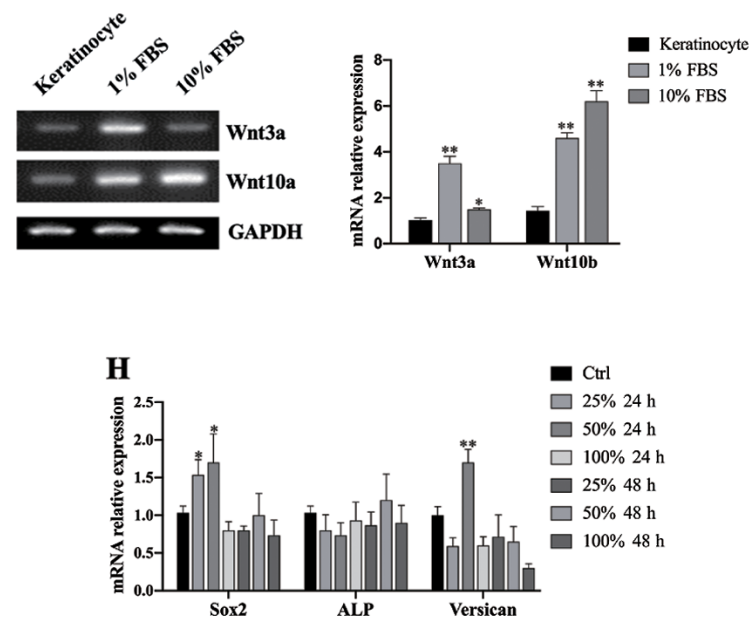

Figure 4. Preparation and evaluation of HaCaT-CM. (A) Morphology of HaCaT cells cultured in 0,1 or $10 \%$ FBS (scale bar, $100 \mu \mathrm{m}$ ). (B) Morphology of keratinocytes isolated from human foreskin (scale bar, $100 \mu \mathrm{m}$ ). (C) RT-PCR analysis of Wnt3a, Wnt10a, Wnt10b, EGF and BMP6 mRNA expression levels in DPCs and HaCaT cells cultured in 0,1 or 10\% FBS. (D) RT-qPCR analysis of Wnt3a, Wnt10a, Wnt10b, EGF and BMP6 mRNA levels in DPCs and HaCaT cells cultured in 0,1 or $10 \%$ FBS; $n=3$. "P<0.05 vs. P5 DPC. (E) RT-PCR analysis of Wnt $3 a$ and Wnt10a mRNA levels in keratinocytes and HaCaT cells cultured in 1 or $10 \%$ FBS. (F) RT-qPCR analysis of Wnt3a and Wnt 10a mRNA levels in keratinocytes and HaCaT cells cultured in 1 or $10 \%$ FBS; $\mathrm{n}=3$. ${ }^{*} \mathrm{P}<0.05$, ${ }^{* *} \mathrm{P}<0.01$ vs. keratinocytes. (G) Cell Counting Kit-8 assay in DPCs cultured with HaCaT-CM under different conditions. (H) RT-qPCR analysis of Sox2, ALP and Versican mRNA levels in DPCs cultured in HaCaT-CM under different conditions; $\mathrm{n}=3$. Data represent the mean \pm SEM. ${ }^{*} \mathrm{P}<0.05$, ${ }^{* *} \mathrm{P}<0.01$ vs. Ctrl. CM, conditioned media; FBS, fetal bovine serum; BMP6, bone morphogenetic protein 6; DPC, dermal papilla cells; ALP, alkaline phosphatase; P, passage; RT-qPCR, reverse transcription-quantitative PCR; Ctrl, control.
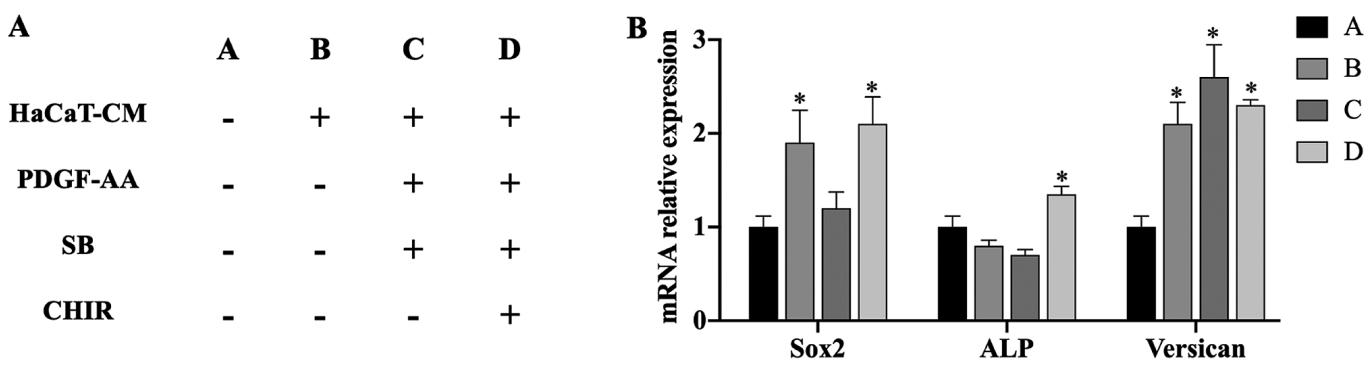

C-1

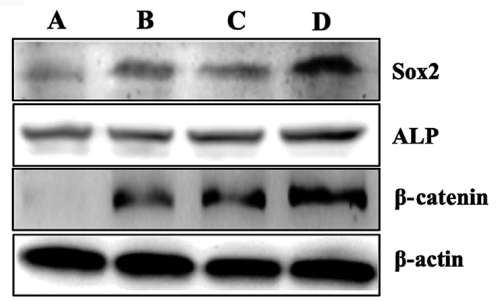

C-2

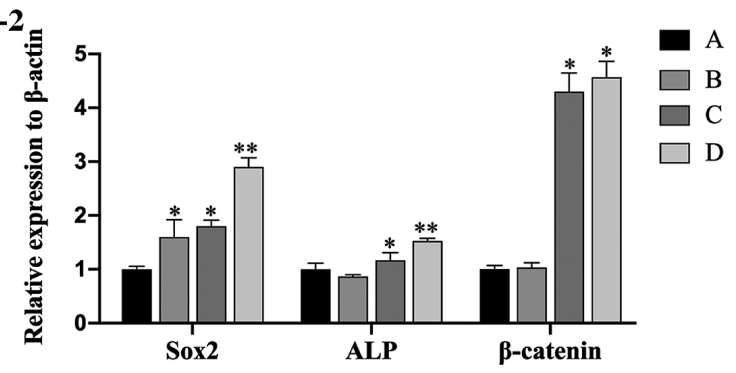

Figure 5. Culture in HaCaT-CM supplemented with SB, CHIR and PDGF-AA enhances the hair-inducing capacity of DPCs. (A) Table showing the small molecule inhibitors used to supplement HaCaT-CM. (B) Reverse transcription-quantitative PCR analysis of Sox2, ALP and Versican mRNA levels in DPCs cultured in HaCaT-CM supplemented with SB, CHIR and PDGF-AA; n=3. (C-1) Western blot analysis of Sox 2, ALP and $\beta$-catenin levels in DPCs cultured in HaCaT-CM supplemented with SB, CHIR and PDGF-AA; (C-2) densitometry analysis; $\mathrm{n}=3$. Data represent the mean \pm SEM. "P<0.05, ${ }^{* *} \mathrm{P}<0.01$ vs. group A. CM, conditioned media; SB, SB431542; CHIR, CHIR99021; PDGF, platelet-derived growth factor; DPC, dermal papilla cells; ALP, alkaline phosphatase. 

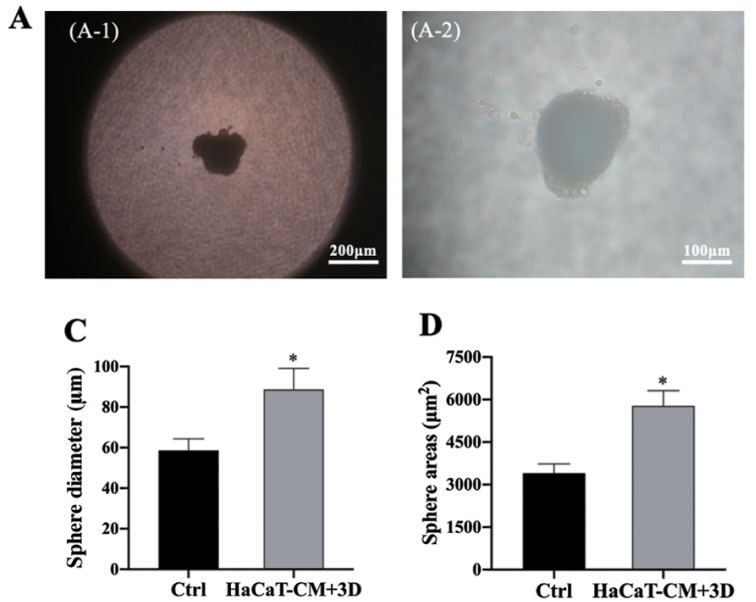

D
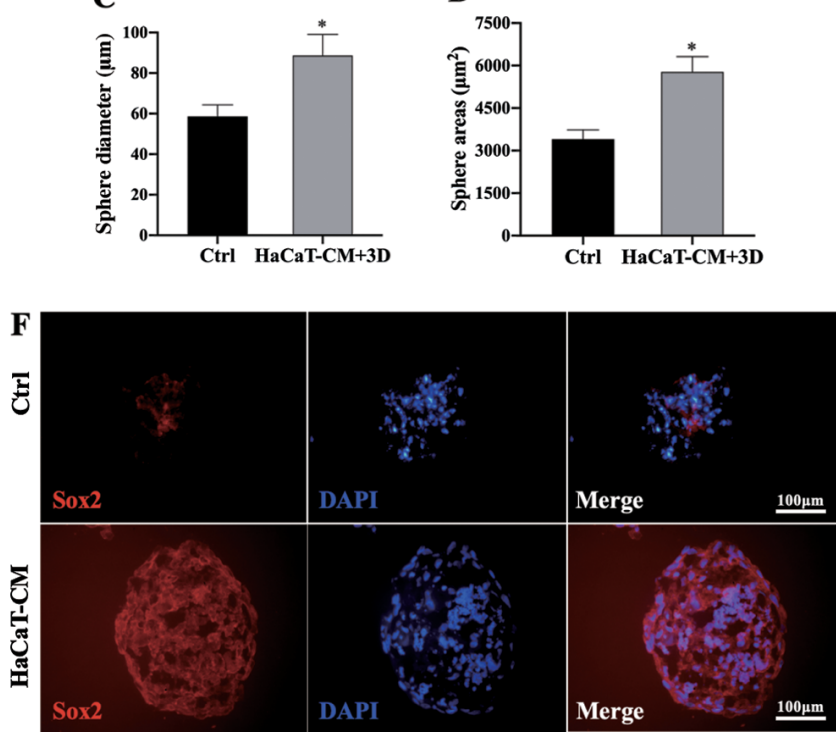

B

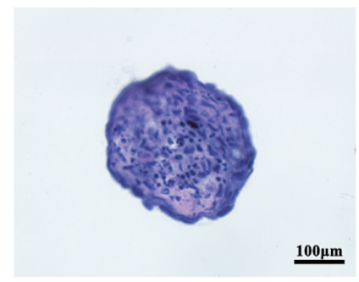

$\mathbf{E}$

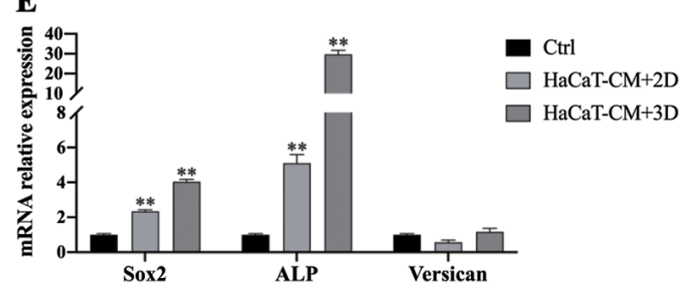

Figure 6. HaCaT-CM supplemented with SB, CHIR and PDGF-AA increases DPC characteristics in a hanging drop culture system. (A) Aggregate of DPCs in hanging drop cultures using HaCaT-CM supplemented with SB, CHIR and PDGF-AA. (A-1) Scale bar, 200 and (A-2) scale bar, $100 \mu \mathrm{m}$. (B) Hematoxylin and eosin staining of DPC spheres (scale bar, $100 \mu \mathrm{m}$ ). (C) Sphere diameter analysis of DPC aggregates cultured in HaCaT-CM supplemented with SB, CHIR and PDGF-AA; n=20. (D) Sphere area analysis of DPC aggregates cultured in HaCaT-CM supplemented with SB, CHIR and PDGF-AA; $n=20$. (E) Reverse transcription-quantitative PCR analysis of Sox2, ALP and Versican mRNA levels in 3D cultures using HaCaT-CM supplemented with SB, CHIR and PDGF-AA, and in two-dimensional cultures in HaCaT-CM supplemented with SB, CHIR and PDGF-AA; $n=3$. Immunocytochemical staining for (F) Sox2 and (G) ALP in DPC spheres from 3D cultures using HaCaT-CM supplemented with SB, CHIR and PDGF-AA (scale bar, $100 \mu \mathrm{m}$ ). Data represent the mean \pm SEM. "P<0.05, ${ }^{* *}$ P<0.01 vs. Ctrl. CM, conditioned media; SB, SB431542; CHIR, CHIR99021; PDGF, platelet-derived growth factor; DPC, dermal papilla cells; ALP, alkaline phosphatase; 3D, three-dimensional; Ctrl, control.

HaCaT-CM supplemented with SB, CHIR and PDGF-AA maintains the hair-inducing capacity of DPCs. To further promote the characteristics of DPCs in vitro and maintain their expression of hair-inducing genes and proteins, $\mathrm{HaCaT}-\mathrm{CM}$ was supplemented with small molecule inhibitors namely $10 \mathrm{mM}$ SB and $3 \mathrm{mM}$ CHIR, as well as $5 \mathrm{ng} / \mathrm{ml}$ PDGF-AA, and then the growth and differentiation of the DPCs at P7 were observed. The medium components in each group are listed in Fig. 5A. The results showed that treatment with $\mathrm{HaCaT}-\mathrm{CM}$ containing SB, CHIR and PDGF-AA significantly upregulated the mRNA expression levels of Sox2, ALP and Versican in DPCs compared with other treatment combinations (Fig. 5B). In addition, a western blot analysis showed that Sox 2, ALP and $\beta$-catenin expression levels were all upregulated after treatment with HaCaT-CM containing SB, CHIR and PDGF-AA compared with other treatment combinations (Fig. 5C).

HaCaT-CM supplemented with SB, CHIR and PDGF-AA increases DPC characteristics in a hanging drop culture system. DPCs at P7 were seeded into hanging drop dishes, and after 7 days of culture, DPC aggregates were observed (Fig. 6A and B). The average diameter and surface area of DPC sphere cultures in HaCaT-CM supplemented with SB, CHIR and PDGF-AA were significantly larger than those cultured in control medium (Fig. 6C and D). Additionally, the expression levels of Sox2 and $A L P$ in DPCs cultured in HaCaT-CM supplemented with SB, CHIR and PDGF-AA in the hanging drop culture were significantly increased compared with those cultured in control medium (Fig. 6E). Moreover, higher expression levels of Sox 2 and ALP were also found by immunofluorescent staining following culture in $\mathrm{HaCaT}-\mathrm{CM}$ supplemented with SB, CHIR and PDGF-AA compared with those cultured in control medium (Fig. 6F and G).

\section{Discussion}

DPs are located in the bulb area of HFs, and it is here that DPCs receive crucial signals from surrounding keratinocytes. Numerous studies have proven that keratinocytes are indispensable cellular components during hair regeneration for their role of interacting with DPCs $(2,6)$. Keratinocytes secrete a variety of bioactive molecules that are necessary for preserving DPC characteristics in vivo $(17,18)$. Accordingly, we speculated that the co-culture of DPCs with keratinocytes or using keratinocyte-CM (containing bioactive molecules) can induce the redifferentiation of DPCs and preserve their hair formation properties after long-term 2D subculture. However, co-cultivation with keratinocytes has some disadvantages, 
including donor-to-donor variability in the characteristics of the keratinocyte cells, and the cells differentiate quickly during cultivation (19), so it is difficult to harvest enough cells to produce high-quality $\mathrm{CM}$ for DPC cultures. As a means of addressing this, HaCaT cells were used in the present study. These cells are a spontaneously immortalized human keratinocyte cell line from adult skin that maintain a stable phenotype during in vitro passaging, which can proliferate in FBS-supplemented medium without the addition of growth factors for continuous growth (19). In the present study, it was found that the mRNA expression levels of Wnt3a and Wnt10b in $\mathrm{HaCaT}$ cells were significantly higher than in primary cultured keratinocytes, indicating a potential ability of these cells to secrete Wnt. Wnt signaling has been demonstrated to regulate HF induction and promote hair growth. The expression of Wnt ligands (Wntla, 3a, 7a, 10b and 11) has also been reported in HFs isolated from postnatal skin, suggesting that $\mathrm{HaCaT}$ cells could be utilized to produce CM for DPC culture (11,20-22). Therefore, unlike normal keratinocytes, a sufficient quantity of CM with stable quality could be collected from the HaCaT cell line for long-term cultivation of DPCs in vitro. To the best of our knowledge, the present study is the first report to explore the $\mathrm{HaCaT}$ cell-CM combined with small molecular compounds to inhibit the dedifferentiation of DPCs in vitro.

The present study examined changes in gene and protein expression levels during 2D cell culture of DPCs. The results showed that there were significant decreases in the levels of Sox2, Versican and ALP during the normal culture (DMEM-F12 with 10\% FBS and $10 \mathrm{ng} / \mathrm{ml}$ bFGF). Furthermore, $\beta$-catenin expression levels were also significantly decreased, indicating that the activity of the $\mathrm{Wnt} / \beta$-catenin pathway is downregulated in DPC cultures. These results are consistent with previous reports $(10,11,14)$, demonstrating that downregulation of the Wnt/ $\beta$-catenin pathway can lead to DPC dedifferentiation.

There are numerous reports that small molecule compounds can be used to inhibit or activate signaling pathways and have been extensively used in numerous cell proliferation and differentiation studies (23-25). For example, recently, Yoshida et al (24) found that treating DPCs with the canonical Wnt/ $\beta$-catenin signaling activator CHIR99021, a potent inhibitor of GSK3 $\alpha$ and GSK3 $\beta$, significantly enhanced the expression of DP signature genes associated with their hair-inducing ability (24). For these reasons, in the present study, DPCs were cultured in the presence of HaCaT-CM supplemented with the Wnt signaling activator CHIR. FGF2/bFGF has also been reported to enhance DPC proliferation (13). Accordingly, in addition to Wnt, bFGF was added as an elemental component in the DPC culture medium.

PDGF is a potent mitogen for cells of mesenchymal origin $(12,13)$. It has been suggested that PDGF-AA expression by immature adipocytes regulates the activity of follicular stem cells and that PDGF receptor $\alpha$ is activated in the DP during the anagen phase (13). Hair reconstitution assays have also revealed that DPCs treated with both PDGF-AA and FGF2 show an improved ability to maintain their hair inductive activity compared with those treated with FGF2 alone (12). PDGF has also been revealed to contribute to the induction and maintenance of the anagen phase in HFs in vivo $(12,13,26)$. Based on these findings, PDGF was considered to be essential factor to promote the growth of DPCs and to maintain hair follicle inductive ability in vivo. For all of these reasons PDGF was also added to the HaCaT-CM in the present study.

During the skin healing process that occurs following skin injury, DPCs dedifferentiate into fibroblast-like cells and participate in the wound healing process. It has been reported that TGF- $\beta 1$ induces DPC dedifferentiation to the fibroblast-like phenotype $(27,28)$. In the present study, it was observed that the expression levels of $\mathrm{p}-\mathrm{Smad} 2$ and $\mathrm{p}-\mathrm{Smad} 3 \mathrm{in}$ the TGF- $\beta /$ Smad signaling pathway gradually increased with passage time, suggesting that TGF- $\beta /$ Smad pathway activation causes DPC dedifferentiation during DPC sub-cultivation. $\mathrm{SB}$ is a potent small molecule inhibitor of the TGF- $\beta / \mathrm{Smad}$ pathway that blocks the type I receptor ALK5 (29). For this reason, $\mathrm{SB}$ was also included in the $\mathrm{CM}$ to inhibit the TGF- $\beta /$ Smad pathways.

Culture of DPCs in HaCaT-CM supplemented with SB, CHIR and PDGF-AA increased the expression levels of Sox2, ALP and $\beta$-catenin more significantly at both the mRNA and protein levels compared with other culture conditions. The mRNA expression levels of Versican were also upregulated 2-fold by culturing in HaCaT-CM supplemented with SB, CHIR and PDGF-AA compared with those in the control medium; however, the protein levels did not show a significant change (data not shown). The reason for this is unclear, but it does suggest that Versican protein expression is inhibited at the post-transcriptional stage through an unknown mechanism. Additionally, the functional role of Versican in hair follicle development and hair growth is also unclear; however, several researchers have suggested that Versican functions as an inhibitor of cell-cell or cell-ECM adhesion $(9,30)$. As DPCs are densely packed in the postnatal skin, Versican could selectively prevent the incorporation of dermal fibroblasts (non-DP-destined cells) into the DP. It is possible that continuous Versican expression in condensed mesenchymal cells may be required to exclude the additional surrounding dermal fibroblasts from condensation, thus maintaining the purity of the induced DPC population (1). We hypothesized that without signals or stimulation from fibroblasts or other dermal components, the Versican levels in DPCs may not rise significantly. However, this will require further investigation to uncover the specific underlying mechanism.

In the hair follicle bulb, DPCs are assembled in a 3D organization, where cell-cell and cell-ECM interactions are crucial for maintaining the biological functions of DPC $(4,5,9,18)$. However, in vitro, 2D cell culture systems do not reflect the in vivo environment and may cause DPCs to rapidly lose their distinctive features and inductive function. Previous studies have reported that in vitro conditions mimicking in vivo-like conditions, specifically DPCs grown into a 3D microtissue, can preserve the natural functions of DPCs and enhance their hair-inducing potential after in vivo transplantation $(31,32)$. In both human and murine hair, the DP size specifies the hair size, shape and cycling (33). Moreover, the DP size mainly depends on DPC number. The average volume of DP in the human scalp is $\sim 536 \times 10^{3}\left(\mu \mathrm{m}^{3}\right)$, and the total number of DPCs within the DP is $\sim 1.3 \times 103$ (34). Accordingly, in the present study, the hanging drop technique was utilized to create cell-aggregated $3 \mathrm{D}$ spheroids. It was found that $3 \times 10^{3}$ cells formed tightly aggregated spheroids in HaCaT-CM supplemented with 
SB, CHIR and PDGF-AA, and that the average volume was $\sim 523 \times 10^{3}\left(\mu \mathrm{m}^{3}\right)$ (data not shown), which was similar to the size of hair follicle DPs in a healthy human scalp. Furthermore, increased Sox 2 and ALP gene and protein expression levels were found in the spheroids formed, indicating that the combination of the hanging drop 3D culture and the supplemented $\mathrm{HaCaT}-\mathrm{CM}$ is favorable for reconstructing an artificial DP structure in vitro. In future studies, we plan to conduct in vivo transplantation to evaluate the hair regenerative capacity of these artificial DP structures using these culture conditions.

To the best of our knowledge, this is the first study to demonstrate the development of a novel DPC culture medium comprising HaCaT-CM supplemented with defined small molecules and growth factors, which has significant synergetic effects in restoring the expression of signature DPC genes. Furthermore, combining this with the hanging drop 3D culture was demonstrated to be more effective in reconstructing DP-like structures with high expression of hair follicle-inducing proteins than $2 \mathrm{D}$ cultures.

In conclusion, cultured and expanded DPCs can change their morphology and lose their hair-inducing ability. However, HaCaT-CM was successfully used to reverse the signature gene expression patterns of high-passage DPCs by culturing in the presence of small molecule inhibitors, including SB, CHIR, and the growth factor PDGF-AA. The present study showed that this culture method could possibly maintain the hair induction ability of DPCs after several passages in expansion cultures. Therefore, this strategy could be used to potentially improve DPC culture methods to provide high-quality and high-quantity DPCs for both the construction of tissue engineered HFs for hair loss treatment and for skin wound repair.

\section{Acknowledgements}

Not applicable.

\section{Funding}

This study was supported by the Projects of International Cooperation and Exchanges Jilin Province (grant no. 20170414058GH), Science and Technology Research Project of the 13th five-year plan of Jilin Province Department of Education (grant no. JJKH20180194KJ) and by the Frontier Interdisciplinary Program of Norman Bethune Health Science Center of Jilin University (grant no. 2013101002).

\section{Availability of data and materials}

The datasets used and/or analyzed during the current study are available from the corresponding author on reasonable request.

\section{Authors' contributions}

GC and YH conceived and designed the experiments. DS and JX performed the experiments. LC, YW and $\mathrm{ZH}$ prepared specimens from the hospital. GC and DS confirmed the authenticity of the raw data and analyzed the results. GC, YH and DS wrote the manuscript. All authors read and approved the final manuscript.

\section{Ethics approval and consent to participate}

The experimental protocol was established according to the ethical guidelines of the Helsinki Declaration and was approved by the Human Ethics Committee of China-Japan Union Hospital of Jilin University (approval no. 2020042606). Written informed consent to participate in this study was provided by the patients or participants' guardians.

\section{Patient consent for publication}

Not applicable.

\section{Competing interests}

The authors declare that they have no competing interests.

\section{References}

1. Xiao SE, Miao Y, Wang J, Jiang W, Fan ZX, Liu XM and Hu ZQ: As a carrier-transporter for hair follicle reconstitution, platelet-rich plasma promotes proliferation and induction of mouse dermal papilla cells. Sci Rep 7: 1125, 2017.

2. Ohyama $M$ and Veraitch O: Strategies to enhance epithelial-mesenchymal interactions for human hair follicle bioengineering. J Dermatol Sci 70: 78-87, 2013.

3. Biernaskie J, Paris M, Morozova O, Fagan BM, Marra M, Pevny L and Miller FD: SKPs derive from hair follicle precursors and exhibit properties of adult dermal stem cells. Cell Stem Cell 5: 610-623, 2009.

4. Wu JJ, Zhu TY, Lu YG, Liu RQ, Mai Y, Cheng B, Lu ZF, Zhong BY and Tang SQ: Hair follicle reformation induced by dermal papilla cells from human scalp skin. Arch Dermatol Res 298: 183-190, 2006.

5. Ohyama M, Kobayashi T, Sasaki T, Shimizu A and Amagai M: Restoration of the intrinsic properties of human dermal papilla in vitro. J Cell Sci 125: 4114-4125, 2012.

6. Higgins CA, Chen JC, Cerise JE, Jahoda CAB and Christiano AM: Microenvironmental reprogramming by three-dimensional culture enables dermal papilla cells to induce de novo human hair-follicle growth. Proc Natl Acad Sci USA 110: 19679-19688, 2013.

7. Driskell RR, Clavel C, Rendl M and Watt FM: Hair follicle dermal papilla cells at a glance. J Cell Sci 124: 1179-1182, 2011.

8. Yang CC and Cotsarelis G: Review of hair follicle dermal cells. J Dermatol Sci 57: 2-11, 2010.

9. Kishimoto J, Ehama R, Wu L, Jiang S, Jiang $\mathrm{N}$ and Burgeson RE: Selective activation of the versican promoter by epithelialmesenchymal interactions during hair follicle development. Proc Natl Acad Sci USA 96: 7336-7341, 1999.

10. Millar SE: Molecular mechanisms regulating hair follicle development. J Invest Dermatol 118: 216-225, 2002.

11. Ouji Y, Nakamura-Uchiyama F and Yoshikawa M: Canonical Wnts, specifically Wnt-10b, show ability to maintain dermal papilla cells. Biochem Biophys Res Commun 438: 493-499, 2013.

12. Rezza A, Sennett R, Tanguy M, Clavel C and Rendl M: PDGF signalling in the dermis and in dermal condensates is dispensable for hair follicle induction and formation. Exp Dermatol 24: 468-470, 2015.

13. Kiso M, Hamazaki TS, Itoh M, Kikuchi S, Nakagawa $H$ and Okochi H: Synergistic effect of PDGF and FGF2 for cell proliferation and hair inductive activity in murine vibrissal dermal papilla in vitro. J Dermatol Sci 79: 110-118, 2015.

14. Gemayel R and Chenette EJ: $\beta$-catenin signalling in dermal papilla cells leads to a hairy situation. FEBS J 283: 2820-2822, 2016.

15. Soma T, Fujiwara S, Shirakata Y, Hashimoto K and Kishimoto J: Hair-inducing ability of human dermal papilla cells cultured under Wnt/ $\beta$-catenin signalling activation. Exp Dermatol 21: 307-309, 2012.

16. Livak KJ and Schmittgen TD: Analysis of relative gene expression data using real-time quantitative PCR and the 2(-Delta Delta C(T)) method. Methods 25: 402-408, 2001. 
17. Moore GP, Du Cros DL, Isaacs K, Pisansarakit P and Wynn PC: Hair growth induction: roles of growth factors. Ann N Y Acad Sci 642: 308-325, 1991.

18. Inamatsu M, Matsuzaki $\mathrm{T}$, Iwanari $\mathrm{H}$ and Yoshizato $\mathrm{K}$ : Establishment of rat dermal papilla cell lines that sustain the potency to induce hair follicles from afollicular skin. J Invest Dermatol 111: 767-775, 1998.

19. Schürer N, Köhne A, Schliep V, Barlag K and Goerz G: Lipid composition and synthesis of HaCaT cells, an immortalized human keratinocyte line, in comparison with normal human adult keratinocytes. Exp Dermatol 2: 179-185, 1993.

20. Dong L, Hao H, Liu J, Tong C, Ti D, Chen D, Chen L, Li M, Liu H, Fu X, et al: Wnt1a maintains characteristics of dermal papilla cells that induce mouse hair regeneration in a $3 \mathrm{D}$ preculture system. J Tissue Eng Regen Med 11: 1479-1489, 2017.

21. Shimizu $\mathrm{H}$ and Morgan BA: Wnt signaling through the $\beta$-catenin pathway is sufficient to maintain, but not restore, anagen-phase characteristics of dermal papilla cells. J Invest Dermatol 122: 239-245, 2004

22. Kishimoto J, Burgeson RE and Morgan BA: Wnt signaling maintains the hair-inducing activity of the dermal papilla. Genes Dev 14: 1181-1185, 2000.

23. Fujimori K, Matsumoto T, Kisa F, Hattori N, Okano H and Akamatsu W: Escape from pluripotency via inhibition of TGF- $\beta$ /BMP and activation of Wnt signaling accelerates differentiation and aging in hPSC progeny cells. Stem Cell Reports 9: 1675-1691, 2017.

24. Yoshida Y, Soma T, Matsuzaki T and Kishimoto J: Wnt activator CHIR99021-stimulated human dermal papilla spheroids contribute to hair follicle formation and production of reconstituted follicle-enriched human skin. Biochem Biophys Res Commun 516: 599-605, 2019.

25. Lu J, Liu H, Huang CT, Chen H, Du Z, Liu Y, Sherafat MA and Zhang SC: Generation of integration-free and region-specific neural progenitors from primate fibroblasts. Cell Rep 3: 1580-1591, 2013.

26. Osada A, Iwabuchi T, Kishimoto J, Hamazaki TS and Okochi H: Long-term culture of mouse vibrissal dermal papilla cells and de novo hair follicle induction. Tissue Eng 13: 975-982, 2007.
27. Bin S, Li HD, Xu YB, Qi SH, Li TZ, Liu XS, Tang JM and Xie JL: BMP-7 attenuates TGF- 31 -induced fibroblast-like differentiation of rat dermal papilla cells. Wound Repair Regen 21: 275-281, 2013

28. Hou-dong L, Bin S, Ying-bin X, Yan S, Shao-hai Q, Tian-zeng L, Xu-sheng L, Jin-ming $\mathrm{T}$ and Ju-lin X: Differentiation of rat dermal papilla cells into fibroblast-like cells induced by transforming growth factor $\beta 1$. J Cutan Med Surg 16: 400-406, 2012.

29. Zheng Y, Zhao YD, Gibbons M, Abramova T, Chu PY, Ash JD, Cunningham JM and Skapek SX: Tgfbeta signaling directly induces Arf promoter remodeling by a mechanism involving Smads 2/3 and p38 MAPK. J Biol Chem 285: 35654-35664, 2010.

30. Lebaron RG: Versican. Perspect Dev Neurobiol 3: 261-271, 1996.

31. Huang YC, Chan CC, Lin WT, Chiu HY, Tsai RY, Tsai TH, Chan JY and Lin SJ: Scalable production of controllable dermal papilla spheroids on PVA surfaces and the effects of spheroid size on hair follicle regeneration. Biomaterials 34 : 442-451, 2013.

32. Tan JJY, Common JE, Wu C, Ho PCL and Kang L: Keratinocytes maintain compartmentalization between dermal papilla and fibroblasts in 3D heterotypic tri-cultures. Cell Prolif 52: e12668, 2019.

33. Chi W, Wu E and Morgan BA: Dermal papilla cell number specifies hair size, shape and cycling and its reduction causes follicular decline. Development 140: 1676-1683, 2013.

34. Elliott K, Stephenson TJ and Messenger AG: Differences in hair follicle dermal papilla volume are due to extracellular matrix volume and cell number: Implications for the control of hair follicle size and androgen responses. J Invest Dermatol 113: 873-877, 1999 .

(i) $($ This work is licensed under a Creative Commons Attribution-NonCommercial-NoDerivatives 4.0 International (CC BY-NC-ND 4.0) License. 